


\section{Rassenbericht Grasland 2018}

Jan Rinze van der Schoot, Henk Schilder

Het Cultuur- en Gebruikswaarde Onderzoek voedergrassen is uitgevoerd door Wageningen Livestock Research in opdracht van en gefinancierd door Plantum.

Wageningen Livestock Research

Wageningen, november 2018

Wageningen Livestock Research

Rapport 1136 
Schoot, J.R. van der, Schilder, H. Rassenbericht Grasland 2018; Wageningen, Wageningen Livestock Research, Rapport 1136.

Samenvatting NL

Rapportage van proefveldresultaten van het rassenonderzoek aan Engels raaigras in de periode 20102017.

Dit rapport is gratis te downloaden op https://doi.org/10.18174/465164 of op www.wur.nl/livestock-research (onder Wageningen Livestock Research publicaties).

(C) 2018 Wageningen Livestock Research

Postbus 338, 6700 AH Wageningen, T 03174839 53, E info.livestockresearch@wur.nl, www.wur.nl/livestock-research. Wageningen Livestock Research is onderdeel van Wageningen University \& Research.

Wageningen Livestock Research aanvaardt geen aansprakelijkheid voor eventuele schade voortvloeiend uit het gebruik van de resultaten van dit onderzoek of de toepassing van de adviezen.

Alle rechten voorbehouden. Niets uit deze uitgave mag worden vermenigvuldigd en/of openbaar gemaakt worden door middel van druk, fotokopie, microfilm of op welke wijze dan ook zonder voorafgaande toestemming van de uitgever of auteur.

Wageningen Livestock Research is NEN-EN-ISO 9001:2015 gecertificeerd. Op al onze onderzoeksopdrachten zijn de Algemene Voorwaarden van de Animal Sciences Group van toepassing. Deze zijn gedeponeerd bij de Arrondissementsrechtbank Zwolle. 


\section{Inleiding}

Dit Rassenbericht Grasland 2018 bevat de nieuwste resultaten van het Cultuur- en Gebruikswaarde Onderzoek (CGO) van Engels raaigras. In het CGO worden rassen van voedergrassen en witte klaver getest op hun landbouwkundige waarde voor de veehouder. Bij goede resultaten komen de rassen op de Aanbevelende Rassenlijst. Voor grasland zijn vooral de eigenschappen standvastigheid, ziekteresistentie (zoals resistentie tegen kroonroest bij Engels raaigras), wintervastheid en drogestofopbrengst belangrijk. De eigenschappen worden beoordeeld op beweidings- en maaiproefvelden, waar de nieuwe rassen samen met de bestaande rassenlijstrassen worden uitgezaaid en onderzocht. 


\section{Resultaten}

Weergegeven zijn de proefveldresultaten van het CGO voor Engels raaigras laat en middentijds doorschietend van de afgelopen jaren. Voor de meeste eigenschappen en de opbrengsten hebben de gegevens betrekking op het gemiddelde van de resultaten verkregen in de periode 2010 t/m 2017 . Vermeld zijn de rassenlijstrassen van de Aanbevelende Rassenlijst Veehouderij 2019 en de rassen in onderzoek van de uitzaaijaren 2014 en 2015. De rassen van 2014 zijn reeds beoordeeld voor opname op Rassenlijst 2019.

In dit Rassenbericht Grasland 2018 zijn van Engels raaigras de nieuwe, aanbevolen rassen van Rassenlijst 2019 vermeld met hun waarderingscijfers. De opname en volgorde is gebaseerd op het advies en het persbericht van de Commissie Samenstelling Aanbevelende Rassenlijst (CSAR).

Voor opname is ook inschrijving in het Nederlands Rassenregister en/of EU-verkeerstoelating nodig. De rassen van 2015 worden volgend jaar beoordeeld, omdat het onderzoek nog niet is afgerond. Voor de overige (gras)soorten die in de Aanbevelende Rassenlijst staan vermeld, zijn geen nieuwe gegevens verkregen. Voor meer informatie over grassoorten, graslandmengsels en raseigenschappen wordt verwezen naar de Aanbevelende Rassenlijst Veehouderij 2019. Ook de eigenschappen van de rassenlijstrassen van de andere grassoorten en typen (Engels raaigras vroeg doorschietend, gekruist raaigras, Italiaans raaigras, timothee en beemdlangbloem) en van witte klaver staan hierin vermeld. 


\section{Toelichting op de tabellen}

Het jaartal achter de rasnaam geeft het eerste jaar van uitzaai en onderzoek aan.

( ) betekent: gegevens zijn gebaseerd op weinig waarnemingen.

- betekent: geen of onvoldoende waarnemingen.

Een hoog cijfer betekent een gunstige waardering van de betrokken eigenschap.

De drogestofopbrengst is per soort of type weergegeven in verhoudingsgetallen. Deze zijn gebaseerd op het gemiddelde van de A- en N-rassen van de desbetreffende soort of het type van de Aanbevelende Rassenlijst 2018. Naast de gemiddelde totale jaaropbrengst van de beweidingsproeven en de maaiproeven is ook de opbrengst van de eerste en tweede snede vermeld. $\mathrm{Bij} 100=\ldots$ ton $\mathrm{ds} / \mathrm{ha}$ is het opbrengstniveau van de rassenproeven vermeld. De opbrengstniveau's van de beweidingsproeven en de maaiproeven zijn niet direct vergelijkbaar door verschillen in beproevingslocaties en management.

De waarderingscijfers en opbrengstgegevens zijn alleen binnen de soort of het type direct vergelijkbaar. Door verschillen in beproevingsperiode, -omvang en -methode kunnen gegevens niet zondermeer gebruikt worden voor een directe onderlinge vergelijking van typen en soorten.

Afkortingen van namen van kwekers of houders/aanvragers kwekersrecht.

AFBI : Agri-Food and Biosciences Institute, Loughgall, Noord Ierland

Bar : Barenbrug Holland B.V., Nijmegen

DLF : DLF A/S, Roskilde, Denemarken

DSV : DSV AG, Lippstadt, Duitsland

IBER : Inst. of Biological Environmental \& Rural Sciences, Aberystwyth, Groot Brittannië

ILVO : ILVO - Plant, Melle, België

NPZ : Norddeutsche Pflanzenzucht Hans-Georg Lembke KG, Holtsee, Duitsland

RAGT : RAGT 2n S.A.S., Rodez, Frankrijk

SdF : Semences de France, Orchies, Frankrijk

Afkortingen van namen van vertegenwoordigers.

$\begin{array}{ll}\text { Bar } & \text { : Barenbrug Holland B.V., Nijmegen } \\ \text { DLF } & \text { : DLF B.V., Kapelle } \\ \text { DSV } & \text { : DSV zaden Nederland B.V., Gennep } \\ \text { Joo } & \text { : Joordens Zaden B.V., Kessel } \\ \text { Lim } & \text { : Limagrain Nederland B.V., Rilland } \\ \text { VDS } & \text { : VANDINTER SEMO BV, Scheemda }\end{array}$




\section{ENGELS RAAIGRAS LAAT DOORSCHIETEND}

Overzicht van de raseigenschappen

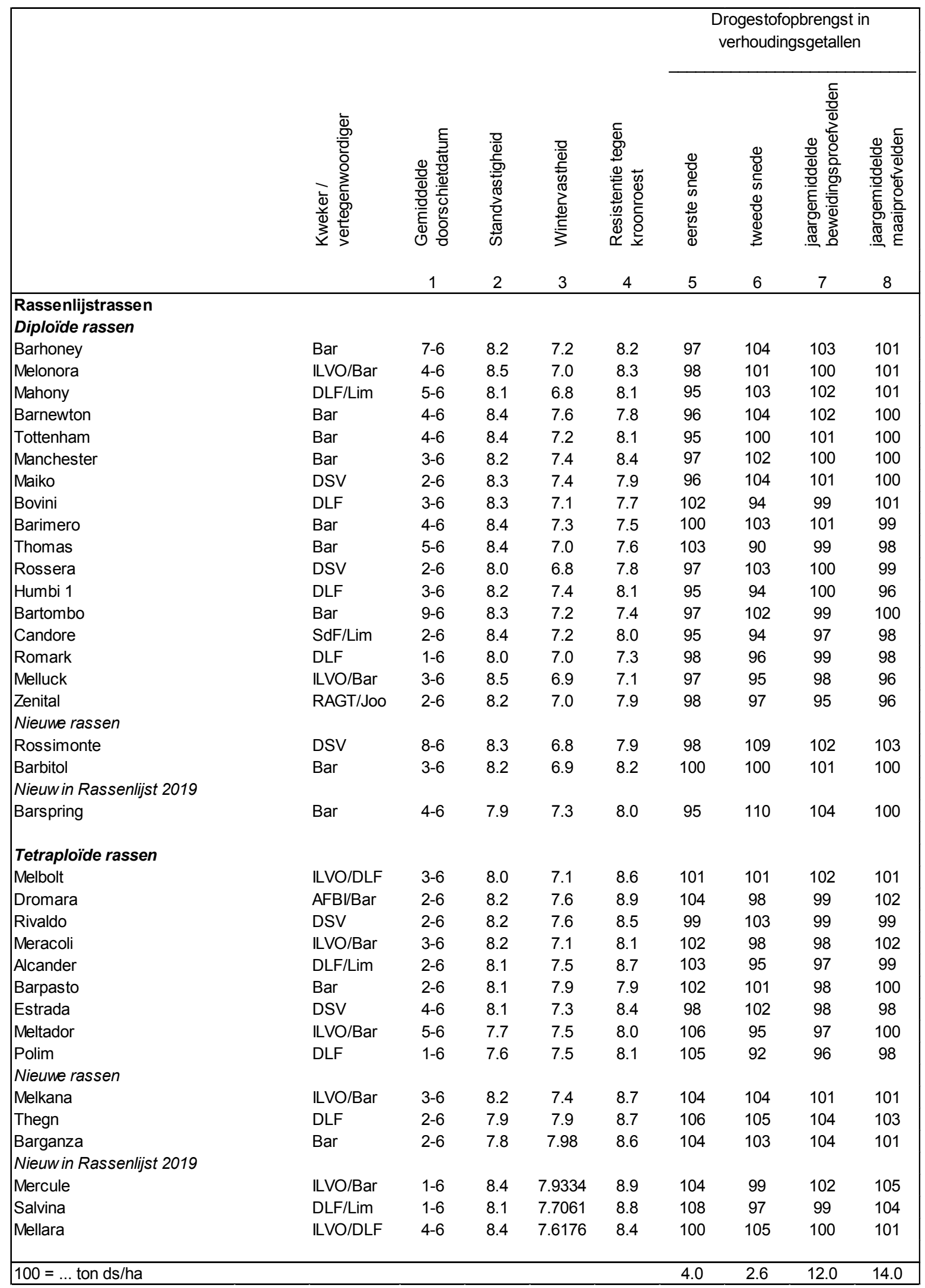




\section{Vervolg ENGELS RAAIGRAS LAAT DOORSCHIETEND}

Overzicht van de raseigenschappen

\begin{tabular}{|c|c|c|c|c|c|c|c|c|c|c|}
\hline & \multirow{3}{*}{ 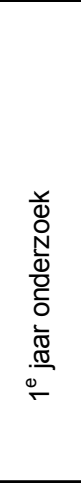 } & \multirow{3}{*}{ 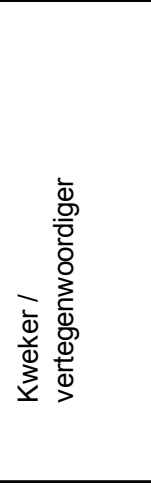 } & \multirow{3}{*}{ 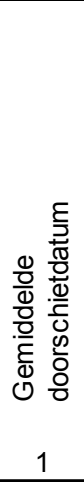 } & \multirow{3}{*}{ 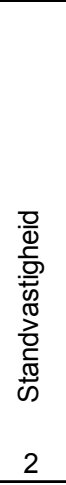 } & \multirow{3}{*}{ 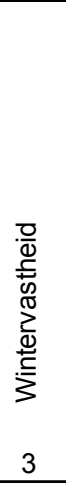 } & \multirow{3}{*}{ 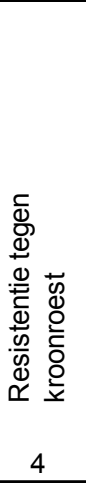 } & \multicolumn{4}{|c|}{$\begin{array}{l}\text { Drogestofopbrengst in } \\
\text { verhoudingsgetallen }\end{array}$} \\
\hline & & & & & & & $\begin{array}{l}\frac{\Phi}{\Phi} \\
\frac{\Phi}{\omega} \\
\Phi \\
\Phi \\
\Phi \\
\Phi\end{array}$ & 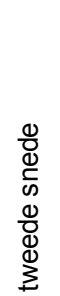 & 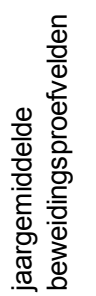 & 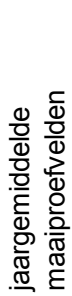 \\
\hline & & & & & & & 5 & 6 & 7 & 8 \\
\hline \multicolumn{11}{|l|}{$\begin{array}{l}\text { Rassen in onderzoek } \\
\text { Diploïde rassen }\end{array}$} \\
\hline Maredo (Elp 062130) & 2014 & DSV & $4-6$ & 8.2 & 7.6 & 7.9 & 94 & 96 & 99 & 97 \\
\hline Andena (Elp 210481) & 2014 & DSV & $30-5$ & 8.1 & 7.2 & 7.9 & 106 & 94 & 101 & 98 \\
\hline 14LPD 113 & 2014 & Bar & $4-6$ & 8.3 & 7.8 & 7.8 & 92 & 105 & 96 & 99 \\
\hline 14LPD 123 & 2014 & Bar & $4-6$ & 7.7 & 7.3 & 7.6 & 92 & 107 & 99 & 97 \\
\hline RGAS974 & 2014 & RAGT/Joo & $31-5$ & 8.5 & 7.3 & 8.5 & 97 & 92 & 97 & 97 \\
\hline Cantik (DT 252) & 2014 & SdF/Lim & $3-6$ & 8.2 & 7.2 & 8.5 & 95 & 92 & 97 & 97 \\
\hline DLF LFD-62760 & 2014 & DLF & $1-6$ & 8.0 & 6.9 & 7.9 & 89 & 97 & 93 & 90 \\
\hline DLF LFD-21352 & 2014 & DLF & $26-5$ & 8.2 & 7.4 & 8.1 & 99 & 95 & 98 & 96 \\
\hline DLF LFD-21385 & 2014 & DLF & $1-6$ & 8.4 & 7.6 & 8.2 & 95 & 101 & 98 & 94 \\
\hline Gauguin (DSVLp 03-654Y) & 2015 & DSV & $31-5$ & 8.0 & 6.8 & 8.2 & 96 & 103 & 100 & 99 \\
\hline Maxwell (DSVLp 090141) & 2015 & DSV & $1-6$ & 7.9 & 7.1 & 7.8 & 97 & 94 & 98 & 98 \\
\hline ILVO 156041 & 2015 & ILVO/Bar & $5-6$ & 8.2 & 7.1 & 8.9 & 94 & 98 & 100 & 98 \\
\hline 15LPD 167 & 2015 & Bar & $31-5$ & 8.6 & 7.5 & 7.5 & 97 & 93 & 93 & 95 \\
\hline 15LPD 137 & 2015 & Bar & $1-6$ & 8.0 & 7.5 & 7.3 & 101 & 93 & 97 & 97 \\
\hline ILVO152964 & 2015 & ILVO/VDS & $3-6$ & 8.4 & 7.5 & 7.1 & 94 & 109 & 104 & 99 \\
\hline DLF LFD-21564 & 2015 & DLF & $3-6$ & 8.2 & 7.5 & 8.5 & 101 & 102 & 103 & 104 \\
\hline DT 333 & 2015 & SdF/VDS & $3-6$ & 8.0 & 6.8 & 7.9 & 91 & 100 & 95 & 97 \\
\hline \multicolumn{11}{|l|}{ Tetraploïde rassen } \\
\hline 13LPT 3083 & 2014 & Bar & $3-6$ & 7.9 & 7.4 & 8.7 & 101 & 95 & 95 & 96 \\
\hline Barwalder (14LPT 224) & 2014 & Bar & $31-5$ & 7.9 & 7.6 & 8.8 & 107 & 94 & 101 & 100 \\
\hline Tanul (DSVLp 03-719) & 2015 & DSV & $2-6$ & 8.3 & 7.5 & 8.0 & 104 & 103 & 99 & 103 \\
\hline |LVO 156038 & 2015 & ILVO/Lim & $1-6$ & 8.3 & 7.5 & 8.9 & 104 & 102 & 103 & 103 \\
\hline DLF LFT-18559 & 2015 & DLF & $30-5$ & 7.6 & 7.1 & 9.3 & 105 & 99 & 94 & 103 \\
\hline Anurad (DLF LFT-41509) & 2015 & DLF & $29-5$ & 7.0 & 7.4 & 8.9 & 118 & 80 & 98 & 96 \\
\hline TT 51 & 2015 & SdF/VDS & $1-6$ & 8.2 & 7.2 & 9.2 & 104 & 92 & 98 & 100 \\
\hline Youpi (TRAS 786) & 2015 & RAGT/Joo & $2-6$ & 8.1 & 7.2 & 8.9 & 103 & 103 & 101 & 102 \\
\hline $100=\ldots$ ton $\mathrm{ds} / \mathrm{ha}$ & & & & & & & 4.0 & 2.6 & 12.0 & 14.0 \\
\hline
\end{tabular}




\section{ENGELS RAAIGRAS MIDDENTIJDS}

Overzicht van de raseigenschappen

\begin{tabular}{|c|c|c|c|c|c|c|c|c|c|}
\hline & \multirow{3}{*}{ 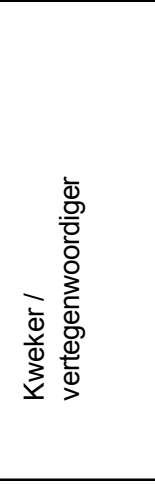 } & \multirow[b]{2}{*}{ 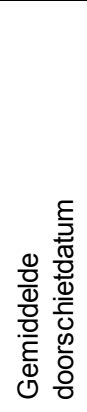 } & \multirow[b]{2}{*}{ 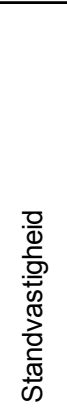 } & \multirow[b]{2}{*}{ 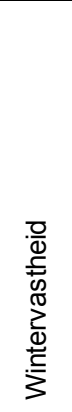 } & \multirow[b]{2}{*}{ 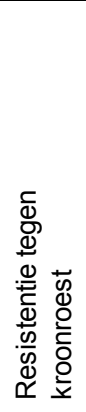 } & \multicolumn{4}{|c|}{$\begin{array}{c}\text { Drogestofopbrengst in } \\
\text { verhoudingsgetallen }\end{array}$} \\
\hline & & & & & & 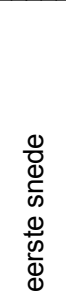 & 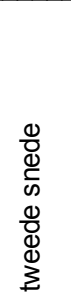 & 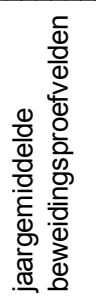 & 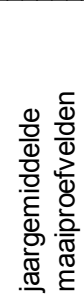 \\
\hline & & 1 & 2 & 3 & 4 & 5 & 6 & 7 & 8 \\
\hline \multicolumn{10}{|l|}{ Rassenlijstrassen } \\
\hline \multicolumn{10}{|l|}{ Diploïde rassen } \\
\hline Sputnik & DLF & $28-5$ & 8.1 & 6.6 & 8.4 & 103 & 100 & 107 & 103 \\
\hline Arelio & DSV & $23-5$ & 8.3 & 7.4 & 7.6 & 104 & 100 & 105 & 102 \\
\hline Melspring & ILVO/Bar & $25-5$ & 8.4 & 6.9 & 8.3 & 103 & 88 & 100 & 101 \\
\hline Arnando & DSV & $29-5$ & 8.4 & 7.5 & 8.0 & 93 & 107 & 101 & 100 \\
\hline Abosan 1 & DLF & $29-5$ & 8.2 & 6.8 & 8.1 & 95 & 98 & 100 & 99 \\
\hline Arsenal & DSV & $22-5$ & 8.2 & 7.1 & 7.6 & 104 & 90 & 100 & 100 \\
\hline Kiani & DLF & $31-5$ & 8.3 & 7.4 & 8.3 & 96 & 100 & 100 & 95 \\
\hline Massimo & DLF/Lim & $24-5$ & 8.2 & 6.6 & 7.9 & 96 & 102 & 98 & 99 \\
\hline Trenio & DSV & $22-5$ & 8.4 & 6.9 & 7.9 & 96 & 100 & 99 & 98 \\
\hline Kaiman & DSV & $30-5$ & 7.9 & 7.1 & 7.8 & 95 & 107 & 99 & 100 \\
\hline Toronto & DSV & $22-5$ & 8.2 & 6.8 & 7.2 & 104 & 87 & 100 & 96 \\
\hline Option & DLF & $25-5$ & 8.2 & 7.0 & 7.9 & 96 & 93 & 98 & 95 \\
\hline Chicago & DSV & $23-5$ & 8.6 & 6.9 & 7.4 & 96 & 95 & 96 & 98 \\
\hline Domiatti 1 & DLF & $29-5$ & 7.9 & 6.5 & 8.0 & 97 & 89 & 96 & 97 \\
\hline \multicolumn{10}{|c|}{ Nieuw in Rassenlijst 2019} \\
\hline Armetto & DSV & $22-5$ & 8.1 & 7.6 & 8.5 & 103 & 96 & 101 & 100 \\
\hline Maqui & DLF & $26-5$ & 8.2 & 7.3 & 8.4 & 99 & 97 & 102 & 98 \\
\hline \multicolumn{10}{|c|}{ Tetraploïde rassen } \\
\hline Diwan & DLF & $27-5$ & 7.9 & 7.6 & 8.7 & 105 & 96 & 101 & 101 \\
\hline Binario & DSV & $22-5$ & 8.0 & 7.7 & 8.7 & 103 & 91 & 100 & 100 \\
\hline Barfamos & Bar & $28-5$ & 8.1 & 7.7 & 8.6 & 96 & 109 & 99 & 100 \\
\hline Barcampo & Bar & $28-5$ & 8.0 & 7.8 & 8.9 & 98 & 99 & 98 & 99 \\
\hline Trivos & DSV & $27-5$ & 7.7 & 7.4 & 8.6 & 101 & 100 & 98 & 101 \\
\hline Maurizio & DSV & $22-5$ & 8.1 & 7.7 & 8.4 & 104 & 96 & 98 & 99 \\
\hline Hurricane & SdF/Lim & $29-5$ & 8.0 & 7.2 & 8.6 & 97 & 103 & 98 & 98 \\
\hline Activa & SdF/VDS & $21-5$ & 7.9 & 7.5 & 8.5 & 103 & 88 & 96 & 97 \\
\hline Melverde & ILVO/Bar & $24-5$ & 8.1 & 7.1 & 8.5 & 95 & 98 & 96 & 99 \\
\hline \multicolumn{10}{|l|}{ Nieuwe rassen } \\
\hline Briant & Bar & $30-5$ & 7.9 & 7.5 & 9.0 & 99 & 105 & 101 & 101 \\
\hline Vifelt & DLF & $24-5$ & 8.0 & 7.6 & 8.7 & 99 & 96 & 100 & 98 \\
\hline Barojet & Bar & $27-5$ & 8.1 & 7.6 & 8.7 & 100 & 109 & 100 & 106 \\
\hline Magena & ILVO/DLF & $31-5$ & 8.3 & 8.1 & 8.6 & 96 & 115 & 103 & 101 \\
\hline Velonit & DLF & $24-5$ & 7.9 & 7.8 & 8.8 & 106 & 96 & 101 & 100 \\
\hline AberGain & IBER/VDS & $28-5$ & 7.8 & 6.9 & 8.5 & 107 & 105 & 99 & 104 \\
\hline \multicolumn{10}{|c|}{ Nieuw in Rassenlijst 2019} \\
\hline Melforce & ILVO/DLF & $28-5$ & 8.3 & 7.9 & 9.0 & 104 & 104 & 108 & 106 \\
\hline Explosion & DSV & $23-5$ & 8.1 & 7.3 & 8.8 & 112 & 89 & 104 & 104 \\
\hline Weldone & DLF/Lim & $29-5$ & 8.2 & 7.7 & 8.8 & 96 & 112 & 102 & 104 \\
\hline Brentford & Bar & $30-5$ & 8.2 & 8.1 & 8.8 & 92 & 114 & 103 & 101 \\
\hline Baramundi & Bar & $29-5$ & 8.1 & 7.7 & 8.7 & 97 & 111 & 102 & 100 \\
\hline Barmigo & Bar & $31-5$ & 7.9 & 7.8 & 8.7 & 97 & 98 & 101 & 102 \\
\hline Boudica & DLF/DLF & $27-5$ & 7.7 & 7.7 & 8.6 & 103 & 95 & 99 & 105 \\
\hline $100=\ldots$ ton $d s / h a$ & & & & & & 4.5 & 2.3 & 12.3 & 14.4 \\
\hline
\end{tabular}




\section{Vervolg ENGELS RAAIGRAS MIDDENTIJDS}

Overzicht van de raseigenschappen

\begin{tabular}{|c|c|c|c|c|c|c|c|c|c|c|}
\hline & \multirow{3}{*}{ 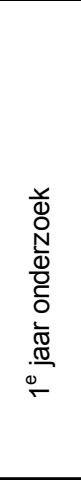 } & \multirow{3}{*}{ 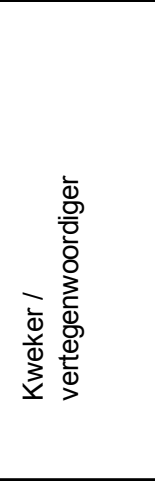 } & \multirow{3}{*}{ 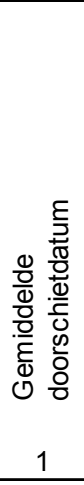 } & \multirow{3}{*}{ 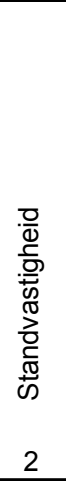 } & \multirow{3}{*}{ 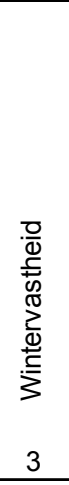 } & \multirow[b]{2}{*}{ 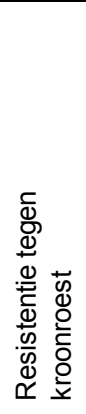 } & \multicolumn{4}{|c|}{$\begin{array}{c}\text { Drogestofopbrengst in } \\
\text { verhoudingsgetallen }\end{array}$} \\
\hline & & & & & & & $\begin{array}{l}\frac{\Phi}{0} \\
\Phi \\
\frac{1}{\omega} \\
\Phi \\
\frac{1}{\omega} \\
\Phi \\
\Phi\end{array}$ & 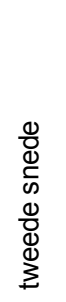 & 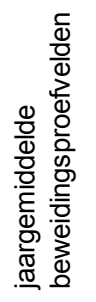 & 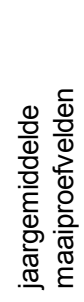 \\
\hline & & & & & & 4 & 5 & 6 & 7 & 8 \\
\hline \multicolumn{11}{|l|}{ Rassen in onderzoek } \\
\hline \multicolumn{11}{|l|}{ Diploïde rassen } \\
\hline RGAS971 & 2014 & RAGT/Joo & $26-5$ & 8.4 & 6.7 & 8.7 & 93 & 96 & 100 & 97 \\
\hline DLF LFD-62637 & 2014 & DLF & $26-5$ & 8.2 & 7.2 & 7.9 & 94 & 97 & 94 & 93 \\
\hline DLF LFD-21397 & 2014 & DLF & $22-5$ & 8.1 & 7.2 & 7.5 & 99 & 92 & 98 & 97 \\
\hline Verdasco (LMG LFD-2061) & 2014 & DLF/Lim & $22-5$ & 7.7 & 7.3 & 7.8 & 104 & 87 & 96 & 97 \\
\hline Aberwolf (Ba14074) & 2014 & IBER/VDS & $23-5$ & 8.3 & 6.1 & 7.7 & 97 & 95 & 97 & 98 \\
\hline Flavinus (DSVLp 072025) & 2015 & DSV & $26-5$ & 7.7 & 7.3 & 8.1 & 102 & 102 & 100 & 101 \\
\hline Iberico (DSVLp 04-738) & 2015 & DSV & $24-5$ & 8.2 & 7.4 & 8.4 & 89 & 96 & 90 & 93 \\
\hline 15LPD 129 & 2015 & Bar & $25-5$ & 8.0 & 7.3 & 8.5 & 100 & 93 & 99 & 97 \\
\hline 15LPD 121 & 2015 & Bar & $25-5$ & 7.8 & 7.4 & 8.1 & 103 & 94 & 103 & 97 \\
\hline 15LPD 110 & 2015 & Bar & $26-5$ & 7.9 & 7.3 & 8.4 & 97 & 94 & 101 & 97 \\
\hline DLF LFD-21534 & 2015 & DLF & $24-5$ & 8.1 & 7.2 & 8.2 & 91 & 100 & 99 & 95 \\
\hline Hannes (DLF LFD-21686) & 2015 & DLF & $26-5$ & 7.5 & 7.4 & 8.2 & 98 & 96 & 101 & 98 \\
\hline Ilea (ILVO156035) & 2015 & ILVO/DLF & $31-5$ & 7.8 & 6.8 & 8.8 & 85 & 117 & 99 & 98 \\
\hline Anterio (LMG LFD-21591) & 2015 & DLF/Lim & $26-5$ & 7.9 & 6.9 & 8.5 & 95 & 109 & 99 & 97 \\
\hline \multicolumn{11}{|l|}{ Tetraploïde rassen } \\
\hline 14LPT 205 & 2014 & Bar & $27-5$ & 8.1 & 7.8 & 9.1 & 99 & 102 & 98 & 102 \\
\hline 14LPT 213 & 2014 & Bar & $31-5$ & 8.0 & 7.6 & 8.8 & 99 & 106 & 103 & 99 \\
\hline Matenga (SLM 24233/04) & 2014 & NPZVDS & $24-5$ & 8.0 & 7.4 & 8.2 & 109 & 91 & 100 & 100 \\
\hline Quadriga (SLM 24215/03) & 2014 & NPZVDS & $29-5$ & 8.1 & 7.1 & 6.8 & 96 & 103 & 96 & 95 \\
\hline TRAH962 & 2014 & RAGT/Joo & $27-5$ & 8.0 & 7.3 & 8.9 & 103 & 96 & 97 & 100 \\
\hline DLF LFT-0808B2 & 2014 & DLF & $21-5$ & 3.3 & 6.5 & 8.6 & 102 & 84 & 80 & 86 \\
\hline Botond (DSVLp 070769) & 2015 & DSV & $22-5$ & 7.9 & 7.5 & 8.3 & 109 & 95 & 99 & 105 \\
\hline 15LPT 240 & 2015 & Bar & $25-5$ & 8.1 & 7.5 & 8.5 & 104 & 94 & 100 & 99 \\
\hline 15LPT 203 & 2015 & Bar & $26-5$ & 8.2 & 7.5 & 8.7 & 103 & 103 & 103 & 101 \\
\hline 15LPT 201 & 2015 & Bar & $25-5$ & 8.0 & 7.5 & 8.4 & 101 & 99 & 98 & 97 \\
\hline Yucatan (LMG LFT-74922) & 2015 & DLF/Lim & $28-5$ & 8.0 & 7.4 & 8.8 & 102 & 96 & 98 & 97 \\
\hline Wappo (DLF LFT-41432) & 2015 & DLF & $25-5$ & 8.2 & 7.5 & 9.0 & 99 & 107 & 105 & 103 \\
\hline Convey (DLF LFT-74918) & 2015 & DLF & $26-5$ & 8.1 & 7.5 & 8.4 & 105 & 95 & 101 & 101 \\
\hline Memisa (LMG LFT-18555) & 2015 & DLF/Lim & $25-5$ & 8.2 & 7.5 & 9.0 & 106 & 91 & 102 & 101 \\
\hline $100=\ldots$ ton ds $/$ ha & & & & & & & 4.5 & 2.3 & 12.3 & 14.4 \\
\hline
\end{tabular}



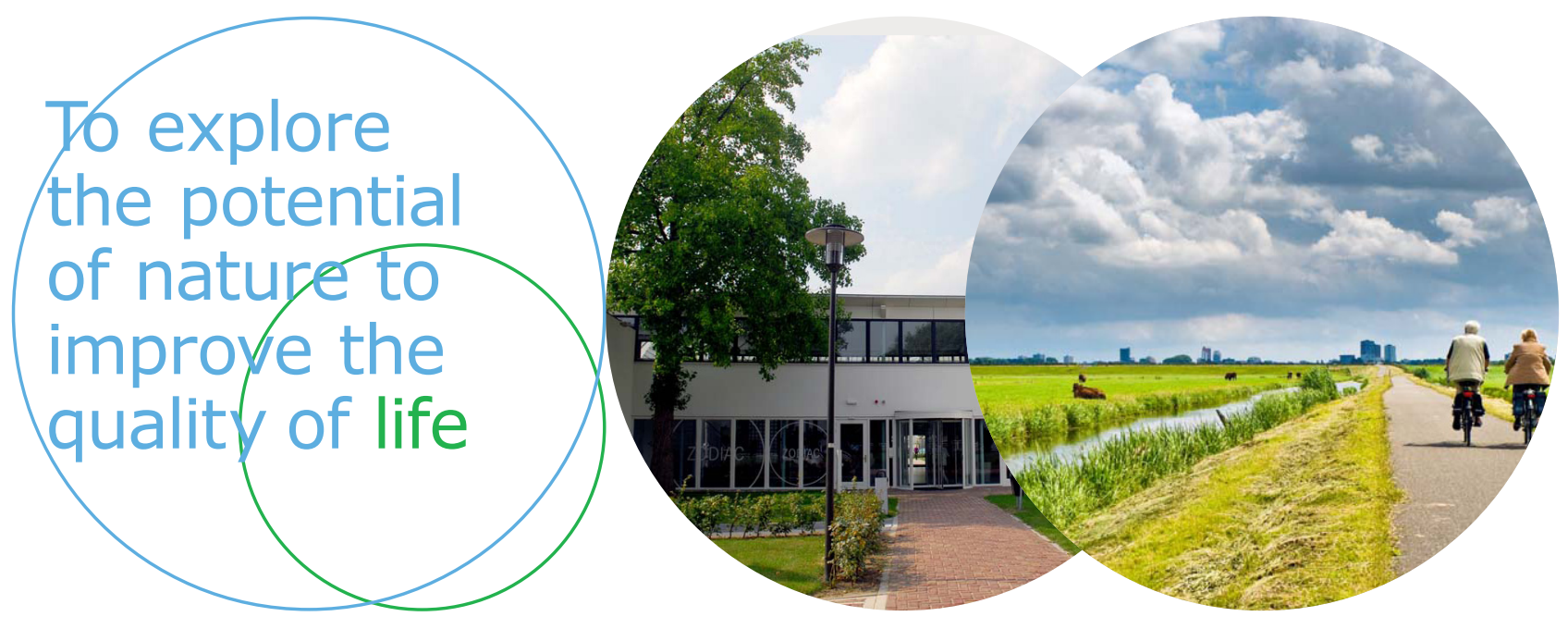

Wageningen Livestock Research Postbus 338

Wageningen Livestock Research ontwikkelt kennis voor een zorgvuldige en $6700 \mathrm{AH}$ Wageningen

T 0317483953

renderende veehouderij, vertaalt deze naar praktijkgerichte oplossingen en innovaties, en zorgt voor doorstroming van deze kennis. Onze wetenschappelijke E info.livestockresearch@wur.nl www.wur.nl/ livestock-research kennis op het gebied van veehouderijsystemen en van voeding, genetica, welzijn en milieu-impact van landbouwhuisdieren integreren we, samen met onze klanten, tot veehouderijconcepten voor de $21 \mathrm{e}$ eeuw.

De missie van Wageningen University \& Research is 'To explore the potential of nature to improve the quality of life'. Binnen Wageningen University \& Research bundelen 9 gespecialiseerde onderzoeksinstituten van Stichting Wageningen Research en Wageningen University hun krachten om bij te dragen aan de oplossing van belangrijke vragen in het domein van gezonde voeding en leefomgeving. Met ongeveer 30 vestigingen, 6.500 medewerkers en 10.000 studenten behoort Wageningen University \& Research wereldwijd tot de aansprekende kennisinstellingen binnen haar domein. De integrale benadering van de vraagstukken en de samenwerking tussen verschillende disciplines vormen het hart van de unieke Wageningen aanpak. 